\title{
On Quantum Computing
}

\author{
An Interview with David Penkler*
}

\author{
by Kemal A. Delic
}

\section{Editor's Introduction}

In recent months, announcements on the progress toward harnessing quantum computing have solicited divers and sometimes strong reactions and opinions from academia and industry. Some say quantum computing is impossible, while others point to actual machines - raising the question as to whether they really are quantum computers. So one sunny morning this past spring I chatted with Dave Penkler to get his view on the present and future of quantum computing. Dave has 40 years of experience with computer hardware and software and has always had a keen interest in their evolution as enabled by the advances in science and technology. Dave is an HP fellow, based in Grenoble, France where his primary interests are in cloud and data-center scale operating systems and networks.

Kemal A. Delic Associate Editor 


\title{
On Quantum Computing
}

\author{
An Interview with David Penkler*
}

\section{by Kemal A. Delic}

Kemal A. Delic: Hello! Good morning.. Thanks for joining us for ACM Ubiquity's breakfast chat.... I am Kemal Delic, associate editor of ACM's Ubiquity Magazine. Today with me I have Dave Penkler; he is an HP Fellow and senior technologist in HP Co. Today. We will chat a little bit about quantum computing. Dave, looking into field of quantum computing-as an extremely interesting domain of scientific research, industrial developments and military uses - how do you see those three fields evolving and developing in the future? Scientific research first...

David Penkler: Good morning Kemal, good to see you again! Yes, on the scientific research front I think Richard Feynman basically set the stage back in 1986 with his keynote about simulating physics with computers-where he essentially puts forward the thesis that in order to simulate quantum phenomena, scale-up required for classical computers would be exponential and that the only feasible way of doing research or studying and simulating quantum phenomena efficiently would be with quantum systems; and I think this is indeed the most promising application of quantum computing in the scientific area. Of course, there are many, many other fields where quantum computing can help move forward science and not least in the area of semiconductor research in fact.

KAD: And how about industrial developments, assuming that those scientific advances interest and research (are) putting the stick in the ground for industrial developments as well?

DP: Indeed, well, leading directly on from the scientific research, the ability to simulating molecules at the quantum level to understand their behavior in pharmaceutical applications as opposed to the classical level, gives us a much more detailed view of the behavior of the molecules. So, for example, in the pharmaceutical industry, they are very interested in being able to get this very detailed simulation of molecules to understand how they behave in different situations.

KAD: I see. And the last thing would be about military uses; assuming that the military has strong interest in this, although lots of advances are not yet in the public view or space. 
DP: Yes, well I think that military is in a way even obliged to very heavily push the research in quantum computing and development of quantum computing because in a way it's like the arms race. Specifically in applications of encryption and decryption of transmitted data, where it has been shown that quantum computers could potentially be used to factor numbers into their prime constituents very quickly and efficiently. If this were in fact to be the case, the military organization that has this capability first will be able to eavesdrop much more effectively on another people's encrypted information.

KAD: I see. And finally, in your view Dave, is quantum computing something that may change the entire technology landscape of $21^{\text {st }}$ century and if so, how?

DP: I don't think it's going to be sort-of a major wave of change. I think starting with scientific research we are going to understand how to build materials that allow us to construct qubits more effectively, to increase the coherence time and so forth; so there will be symbiotic relationship between the research and industry for quite some time. But I think classical computing will still be a mainstay of computing for the next 20 to 30 years, for example, payroll and all this discrete stuff will still be done better on classical computers, because quantum computers are intrinsically probabilistic. However there are they are certain algorithms like some search algorithms or using flux qubits. A recent commercial venture into quantum computers can solve optimization problems, using Grover's algorithm they can do some very efficient searching in a very, very large spaces-so, I think quantum computers will be embedded into classical systems as accelerators with quantum algorithms there, where they do things best.

KAD: Thanks Dave! We appreciate you addressing quantum computing developments. Thank you for listening to this Ubiquity podcast. I am Kemal Delic and that was our conversation with Dave Penkler, fellow in Hewlett-Packard Co. Thanks!

*This interview has been edited for print.

\begin{abstract}
About the Author
Kemal A. Delic is a senior technologist with Hewlett-Packard Co. He is also an Adjunct Professor at PMF University in Grenoble, Advisor to the European Commission FET 2007-2013 Programme and Expert Evaluator for Horizon 2020. He can be found on Twitter @OneDelic.
\end{abstract}

DOI: $10.1145 / 2817212$ 